\title{
Primary Inguinal Hernia Repair Using TAPP with External Fixation (Abdelhamid Technique) Versus Prolene Hernia System
}

\author{
Mohamed Salah Abdelhamid ${ }^{1}$, Tamer Mohamed Nabil ${ }^{1}$, Hesham Ahmed Nafady ${ }^{1}$, \\ Amr Mohamed Ali ${ }^{1}$, Ahmed Mohamed Rashad ${ }^{1}$, Seham Anwar Emam ${ }^{1}$, Ibrahim Said Abdelaziz ${ }^{1}$, \\ Tamer Elgaabary ${ }^{2}$
}

${ }^{1}$ Surgery Department, Bani-Suef Faculty of Medicine, Bani-Suef University, Bani-Suef, Egypt

${ }^{2}$ Surgery Department, Fayum Faculty of Medicine, Fayum University, Fayum, Egypt

\section{Email address:}

mohamedsalah2000@hotmail.com (M. S. Abdelhamid), tamernbl@gmail.com (T. M. Nabil), heshamahmednafady@yahoo.com (H. A. Nafady), Amrbekheet@gmail.com (A. M. Ali), Rashad7793@yahoo.com (A. M. Rashad), Sihamanwer8@gmail.com (S. A. Emam), Ibrahim sayed@hotmail.com (I. S. Abdelaziz), drtamerelgaabary@hotmail.com (T. Elgaabary)

\section{To cite this article:}

Mohamed Salah Abdelhamid, Tamer Mohamed Nabil, Hesham Ahmed Nafady, Amr Mohamed Ali, Ahmed Mohamed Rashad, Seham Anwar Emam, Ibrahim Said Abdelaziz, Tamer Elgaabary. Primary Inguinal Hernia Repair Using TAPP with External Fixation (Abdelhamid Technique) Versus Prolene Hernia System. Advances in Surgical Sciences. Vol. 5, No. 3, 2017, pp. 36-40.

doi: $10.11648 /$ j.ass.20170503.12

Received: April 8, 2017; Accepted: April 19, 2017; Published: May 31, 2017

\begin{abstract}
Purpose To asses TAPP with external fixation against PHS and to find which is more suitable in primary inguinal hernia repair. Inguinal hernia repair is the most frequently performed operation in general surgery and so even modest improvements in clinical outcomes are important. The most important criteria for the choice of method are safety (morbidity and mortality), recurrence rates and convenience for the patient, including the risk of chronic groin pain. In recent years, great importance has been placed on the short-term comfort of the patient, measured by post operative pain and the length of convalescence. This is a retrospective study for 250 patients done years ago at Al Hayat Hospital Jeddah, KSA, Bani-Suif University Hospital and Bani Suif Health Insurance Hospital. The study design and data collection were between $10-2015$ and 10 - 2016. We reported the patients for duration of operation, hospital stay, cost, recurrence, and return to work. The mean operative time in TAPP with external fixation was 70 min while in PHS was 55 min. The cost was less in PHS while long of stay [LOS] was the same. Recurrence was $1.5 \%$ in TAPP with external fixation on the other hand it was $4 \%$ in PHS. Patients returned back to work after 5 days in TAPP with external fixation and after 9 days in PHS. It is to be concluded that TAPP with external fixation is associated with longer operative time and higher cost but the incidence of recurrence is much less while return to work was earlier in TAPP with external fixation advocating it to be used as a primary option in primary inguinal hernia repair.
\end{abstract}

Keywords: Inguinal, TAPP, PHS, External Fixation

\section{Introduction}

3500yrs ago, Egyptian physicians reported the management of hernia by conservative means that included the snuggly fitting bandage for reduction and support. For 100 years the Bassini-type repair for inguinal hernia was the standard method. The Lichtenstein "tension free" mesh repair replaced it on the grounds of much lower recurrence rates,
$<5 \%$ vs. $\sim 15 \%$. However, open procedures all have significant long-term discomfort rates of up to $53 \%$. Laparoscopic repair has become a genuine option in the last 15 years and offers low recurrence $(<1 \%)$ and minimal longterm discomfort.

However, it has not been widely taken up [1].

Inguinal hernia repair is the most frequently performed operation in general surgery and so even modest improvements in clinical outcomes are important. The most 
important criteria for the choice of method are safety (morbidity and mortality), recurrence rates and convenience for the patient, including the risk of chronic groin pain. In recent years, great importance has been placed on the shortterm comfort of the patient, measured by post operative pain and the length of convalescence [2].

The myopectineal orifice is the area in the groin bounded superiorly by the internal oblique muscle and the transverse abdominal muscle, laterally by the iliopsoas muscle, medially by the rectus muscle and sheath, and inferiorly by the pecten of the pubis. This bony muscular framework is bridged and bisected by the inguinal ligament, transversed by the spermatic cord and femoral vessels, and sealed like a drum on its inner surface by the transversalis (endopelvic) fascia only. Therefore, the integrity of the myopectineal orifice is dependent on the transversalis fascia [3]

Laparoscopic inguinal hernia repair [LIHR] was first described in 1990 byGer, who placed a simple mesh plug in the defect [4]. LIHR yields comparable results to open hernia repair [OHR].

Many studies have shown that LIHR gives similar results in terms of recurrence as OHR but with the added advantage of reduced post operative pain and wound infection, and an earlier return to activity [5]. Several laparoscopic procedures have successfully passed the stage of feasibility assessment and are currently under scrutiny with regard to indications. LIHR is a typical example of such investigations [6].

It is not necessary to secure the mesh during laparoscopic TAPP inguinal hernia repair from the interior and it is fixed only to the exterior allowing a reduction in size of the ports and considerable reduction in cost with less post TAPP pain with mild increase in operative time. [7]

Olmi et al. [8] stated that their experience demonstrates that fibrin glue (Tissocol) is an effective method for mesh fixation during TAPP. Kapiris et al. [9] stated that TAPP repair is a technically demanding laparoscopic technique but, once mastered, is safe and effective with a high degree of patient satisfaction. Stapling the mesh is not necessary in most cases, thus resulting in remarkably low costs.. External fixation is associated with less pain and less analgesics; this is because the use of a 12-mm port is omitted, and the possibility of nerve entrapment is also abolished as no tuckers or staplers are used [10]. The issue of mesh fixation in LIHR remains unsolved. The need to fix the mesh arises from the fear of increasing recurrence rates. However, specific complications have emerged as a result of mesh fixation. Avoiding routine fixation of the mesh helps to decrease complications and operative costs without increasing recurrence rates. [9].

Among the several techniques for the treatment of inguinal hernia, the Prolene Hernia System (P. H. S.) has gained widespread acceptance over the past few years. This is an original prosthetic device that combines, in a single step, what other techniques offer separately, namely an underlay patch (preperitoneal placement), an onlay patch (subfascial placement) and a connector that joints them together and works as a plug (10). The PHS has been developed for the tension-free repair of inguinal hernias. It provides 3 components of the most popular mesh devices is use today for open hernia repairs, in a single, easily used device. The 3 components include:

1. An underlay mesh similar to that used in Gilbert's sutureless repair [11].

2. An onlay mesh similar to that used in Lichtenstein repair [12].

3. A connecting cylinder between the two; not as bulky as the plug described by Rutkow, and not as hard as the rolled plug described by Lichtenstein [13].

\section{Patients}

This is a retrospective study for 250 patients done years ago at Al Hayat Hospital Jeddah, KSA, Bani Suif University Hospital and Bani Suif Health Insurance Hospital. The study design and data collection were between 10 -2015 and 10 2016. Included in the study were patients with unilateral inguinal hernia both direct and indirect. Both bilateral and recurrent hernias were excluded from the study. There were 200 patients with inguinal hernias went TAPP with external fixation and 50 patients with inguinal hernias went repair with PHS. We reported the patients for duration of operation, hospital stay, cost, recurrence, and return to work.

\section{Methods}

All patients received antithrombotic prophylaxis in the form of compression stockings, subcutaneous LMWH. All patients were performed under general anesthesia. At induction of anesthesia all patients received antibiotic prophylaxis.

\subsection{TAPP with External Fixation}

\subsubsection{Pneumoperitoneum}

A pneumoperitoneum is created using a Verres needle, and an intra-abdominal pressure of $15 \mathrm{~mm} \mathrm{Hg}$ is maintained. The $10 \mathrm{~mm}$ trocar in infra-umbilical position is inserted. The telescope is then inserted and the intraabdominal cavity explored. Two $5 \mathrm{~mm}$ trocars are inserted lateral to each rectus muscle, at the same level as the umbilical trocar.

\subsubsection{Peritoneal Flap}

Creating the peritoneal flap the repair is initiated. The laparoscope is pointed toward the afflicted inguinal canal. The peritoneal defect or hernia is identified. The other inguinal canal is inspected. If an asymptomatic hernia sac is found on the other side, the patient is excluded from the study. The lateral umbilical ligament is located as well as the inferior epigastric artery and vein. A peritoneal incision is. The incision is extended from the lateral aspect of the inguinal region to the lateral umbilical ligament as high as possible to maximize exposure of the region.

\subsubsection{Landmarks}

Exposing the inguinal structures Cooper's ligament is 
exposed as well as the inferior epigastric vessels and the spermatic cord. It is essential to expose the uncovered abdominal wall meticulously (without peritoneum) and remove all fatty layers.

\subsubsection{Dissecting the Hernia Sac}

Dissecting the hernia sac the indirect inguinal hernia sac should be dissected carefully from the spermatic cord.

Particular care should be taken not to dissect lateral and inferior to Cooper's ligament, as the iliac artery and vein will enter the femoral canal at this site.

\subsubsection{Mesh Insertion}

Inserting and anchoring the mesh before inserting the mesh, its mid-point is anchored bilaterally to two prolene threads that are tied with the knots towards the back, to be facing the abdominal wall when it is pulled using the port closure device. The threads are pulled, first obliquely just medial to the anterior iliac spine, second lateral to midline, pulled then tied in place.

\subsubsection{Peritoneal Closure}

Closing the peritoneum the peritoneum is closed while reducing the pressure with vicryl $2 / 0$. For post operative pain relief, injection of diclofenac sodium $75 \mathrm{mg}$ i.m. is given post-operatively in the recovery room to all patients [14].

\subsection{PHS}

Using standard sterile surgical procedures the skin was prepared with povidone-iodine solution. An oblique $4-5 \mathrm{~cm}$ skin incision was made in the inguinal region, and the inguinal canal was opened in layers. Indirect inguinal hernias are repaired by first dissecting the sac from the cord structures back to the internal ring. The sac then ligated and divided. The underlay mesh is then folded, placed and made to expand so that it lay flat in the preperitoneal space under the transversalis fascia. The cylinder lies in the internal ring adding the security of a plug to the underlay mesh to prevent recurrence. Overlay patch is modified to accommodate cord structures. The cord structures, after exiting the internal ring, lie on top of the overlay mesh where it covers the floor of the canal. The external oblique fibers are then closed over the cord structures and overlay mesh. Repair of direct inguinal hernias requires circumscribing the floor of the canal around the defect. Operating through the defect, space is then created between the floor of the canal and the peritoneum (actualization of the preperitoneal space). The underlay mesh is the placed through the defect and expanded under the floor of the canal in the preperitoneal space. The cylinder lies in the defect for additional protection from recurrence. The overlay mesh is then placed down over the floor of the canal and modified to accommodate the cord structures. Cord structures are allowed to lay on the overlay mesh. The overlay mesh sutured around the floor of the canal. The external oblique is then closed over the cord structures and overlay mesh. When the internal ring was not dilated the transversalis fascia covering the posterior wall of the inguinal canal was cut open for a length of $2 \mathrm{~cm}$. With blunt dissection using a finger and gauze, the preperitoneal space of Bogros was dissected out to create a plane for the underlay part of the mesh. The underlay patch was spread out in the preperitoneal space created, and the defect in the transversalis fascia was narrowed with one or two interrupted sutures of 2-0 polypropylene [15].

\section{Results}

Table 1. Abdelhamid technique versus PHS.

\begin{tabular}{lll}
\hline Mean of & TAPP with external fixation & PHS \\
\hline $\begin{array}{l}\text { Duration of operation in } \\
\text { minutes }\end{array}$ & 70 & 55 \\
Cost in \$ & 1800 & 1500 \\
Hospital stay in nights & 1 & 1 \\
Recurrence & $3 / 2001.5 \%$ & $2 / 504 \%$ \\
Return to work in days & 5 & 9 \\
\hline
\end{tabular}

\section{Discussion}

The PHS is constructed of high-porosity polypropylene for optimum tissue ingrowth. It can be used to repair all types of inguinal hernias, including femoral hernias. The PHS has many theoretical advantages over the other conventional forms of repair. It provides a larger allowable surface for effective tissue ingrowth and fibrosis. The underlay patch lies in the preperitoneal space and provides a double-layered reconstruction of the transversalis fascia. The PHS protects both the femoral and inguinal region from recurrence. The underlay components secures the myopectineal orifice and the onlay component secures the posterior wall of the inguinal canal. Placement of the underlay component in the preperitoneal plane has theoretical advantages. It employs Pascal's principle of hydrostatic pressure to allow the intraabdominal pressure to keep the mesh secure in place. It has all the advantages of a secure posterior repair from a simple anterior approach. It is a versatile prosthesis that can be used for other abdominal wall hernia repairs [15].

Mechanisms of recurrence may be related to technical difficulties, and the use of inadequate mesh size and positioning [16]. Any model should take into account the additional equipment costs versus time costs related to laparoscopic surgery, as patients return to work earlier.

Unfortunately, such analysis has shown that an early return to work does not offset the additional costs associated with laparoscopic hernia repair [17]. Reliable laparoscopic fixation of meshes prior to their fibrous incorporation is intended to minimize recurrence following TAPP [18].

As all our operations done through the same team under the same circumstances with the same facilities comparison between the TAPP with external fixation (Abdelhamid technique) versus PHS could come to a faire out come. Regarding the length of operation, the mean time of TAPP with external fixation of the mesh was 70 minutes while that for PHS was 55 minutes, this is in favors of PHS especially that most of the patients subjected for hernia repair almost elderly, so it is not preferable to be subjected to long time of 
anesthesia with abdominal insufflations in case of TAPP

Cost of operation is of utmost importance especially in developing countries, the cost for TAPP with external fixation (Abdelhamid technique) after omitting the use of a $12 \mathrm{~mm}$ trocar and the use of a fixing device (tuckar or stapler) still higher than PHS (1800\$ against 1500\$). Although in our technique there is much reduction in cost comparing it with traditional TAPP with mesh fixation [19].

Fibrin glue (Tissocol) is an effective method for mesh fixation during TAPP [20]. External fixation has the advantage of being fixed well. TAPP repair is a technically demanding laparoscopic technique, but once mastered, is safe and effective with a high degree of patient satisfaction [21]. By all criteria of success - recurrence, recovery, long term symptoms and economics-LIHR using our method, is the winner. In our study we omitted the use of the disposable 12 $\mathrm{mm}$ trocar and the use of any hernia tucker, which are the most expensive components in the cost of TAPP hernia repair(14) Stapling the mesh is not necessary in most cases, thus resulting in a remarkably low cost. Again external fixation of the mesh is superior as it is associated with fixation and at the same time low cost. [19]

Long of stay was the same for TAPP with external fixation (A bdelhamid technique )and in PHS, but return to work was much higher in PHS as it was 9 days compared to 5 dayes in our technique.This in favor of our technique and in general for TAPP

A model that takes into account the additional equipment cost and time cost related to laparoscopic surgery, as patients return to work earlier. Unfortunately, this analysis showed that an early return to work does not offset the additional costs associated with laparoscopic hernia repair [22].

One of the most important factors in hernia surgery is defining recurrence rate. In our technique it was $1.5 \%$ versus $4 \%$ in PHS, this is in favor of out technique.

Olmi et al. [8] stated that their experience demonstrates that fibrin glue (Tissocol) is an effective method for mesh fixation during TAPP, yet our method has the advantage that the mesh is fixed well to the anterior abdominal wall from the inside. Moreover, the mesh can be manipulated to change its position, in contrast to glue, which, once fixed, cannot be manipulated. Also, some degree of tension can be applied to our mesh.

It is to be concluded that TAPP with external fixation is associated with longer operative time and higher cost but the incidence of recurrence is much less while return to work was earlier in TAPP with external fixation advocating to be used as a primary option in primary inguinal hernia repair

\section{References}

[1] Wall ML, CherianTH and. Lotz JC (2008) Laparoscopic Hernia Repair-The Best Option?" Acta Chirurgica Belgica, 108: 186-191.

[2] Poobalan AS, Bruce J, King PM (2001) Chronic pain and quality of life following open inguinal hernia repair. Br J Surg;

\section{8: $1122-1126$.}

[3] Wantz GE (1999) Abdominal wall hernias. In Schwartz SI (ed). Principles of Surgery, $6^{\text {th }}$ ed.: Mc Grow-Hill, Inc., New York; pp 1541-44.

[4] Neugebauer EAM, Troidi H, Kum CK (2006) The EAES clinical practice guidelines on laparoscopic cholecystectomy, appendectomy and hernia repair. In: Neugebauer EAM, Sauerland S, Fingerhut A, Millat B, Buess G (eds) EAES guidelines for endoscopic surgery. Springer, Berlin, pp 265289.

[5] Koniger J, Redecke J, Butters M (2004) Chronic pain after hernia repair: a randomized trial comparing Shouldice, Lichtenstein and TAPP. Langens Arch Surg 389:361-365.

[6] Tatulli F, Chetta G, Caputi A, Mastrototaro P, Ruggieri T (2009) Laparoscopic inguinal hernia repair: audit of our experience with laparoscopic trans-abdominal pro-peritoneal repair (TAPP) Chir Ital 61(1):47-53.

[7] Abdelhamid MS, Sadat AM, Abdelhakim AF, Nabil TM, Abdelbasset MS, Bechet AMA, Nafady HA, Shawky KA (2013) Transabdominal Pre-Peritoneal Mesh for Inguinal Hernia Repair with External Fixation versus Mesh Stapling Surgical science 4: 516-519.

[8] Olmi S, Erba L, Bertolini A, Scaini A (2006) Fibrin glue for mesh fixation in laparoscopic transabdominal preperitoneal (TAPP) hernia repair indications, technique and outcomes. Surg Endosc 20(12):1846-1850.

[9] Kapiris S, Mavromatis T, Andrikopoulus S, Georgiades C, Floros D, Diamantoupolus G (2009) Laparoscopic transabdominal preperitoneal hernia repair (TAPP): stapling the mesh is not mandatory. J Laparosc Adv Surg Tech 19(3):419-422.

[10] Licheris, Erdas E, Martinasco L, (2004) Treatment of inguinal hernia with PHS. Chir Ital 56 (1): 127-34.

[11] Gilbert Al, Graham MF (1995) Improved sutureless technique - advice to experts. Problems in General surgery 12: 117-9.

[12] Lichtenstein IL, Shulman Ag, Amid PK, Montllor MM (1989) The tension-free hernioplasty. Am J Surg 157: 188-93.

[13] Rutkow IM, Robbins AW (1993) Tension-free inguinal hernioplasty: a preliminary report on the mesh plug technique. Surgery; 114: 308-312.

[14] Abdelhamid MS (2011) Transabdominal pre-peritoneal inguinal hernia repair with external fixation Hernia 15:185188 .

[15] Chandiramani VA, Katara AW, Pandyas M, et al (2003): PHS in the tension free repair of primary inguinal hernias. Ind $J$. Surg 65 (6): 488-491.

[16] Watson A, Ziprin P, Chadwick S (2006) TAPP repair for inguinal hernias - a new composite mesh technique. Ann R Coll Surg Engl 88(7):678-681.

[17] Voyles CR, Hamilton BJ, Johnson WD, Kano N (2002) Metaanalysis of laparoscopic inguinal hernia trials favors open hernia repair with preperitoneal prosthesis. Am J Surg 184:6-10.

[18] Schwab R, Schumacher O, Junge K, Binnebosel M, Klinge U, Becker HF (2008) Biomechanical analyses of mesh fixation in TAPP and TEP hernia repair. Surg Endosc 22(3):731-738. 
(Abdelhamid Technique) Versus Prolene Hernia System

[19] AbdelhamidMS, Abouleid AH, Abdelmola MH, Rashad AM, Negida MA, Gharib AZ (2016) Patients Satisfaction Post Laparoscopic TransabdominalPreperitoneal Inguinal Hernia Repair with External FixationVersus Post Internal Fixation. Journal of Surgery 4(2): 20-23.

[20] Olmi S, Erba L, Bertolini A, Scaini A, (2006) Fibrin glue for mesh fixation in laparoscopic transabdominal preperitoneal (TAPP) hernia repair indications, technique and outcomes. Surgical Endoscopy volume 20 (12): 1846-1850.
[21] Kapiris S, Mavromatis T, Andrikopoulus S, Georgiades C, Floros D, Diamantoupolus G, (2009) Laparoscopic Transabdominal Preperitoneal Hernia Repair (TAPP): Staplingthe Mesh Is Not Mandatory. Journal of Laparoscopic \& Advanced Surgical Techniques 19 (3): 419-422.

[22] Voyles CR, Hamilton BJ, Johnson WD, Kano N (2002) Metaanalysis of laparoscopic inguinal hernia trials favors open hernia repair with preperitoneal prosthesis. Am J Surg 184:610 . 